\title{
Does Ramadan fasting affect sleep?
}

\author{
A. BAHAMMAM \\ Sleep Disorders Center, College of Medicine, King Saud University, Riyadh, Saudi Arabia
}

\section{SUMMARY}

Experimental fasting has been shown to alter the sleepwakefulness pattern in various species. As fasting during Ramadan is distinct from experimental fasting, the physiological and behavioural changes occurring during Ramadan fasting may differ from those occurring during experimental fasting. There has been increased interest in recent years in sleep changes and daytime sleepiness during Ramadan. Moreover, many of those who fast during Ramadan associate this fasting with increased daytime sleepiness and decreased performance. This raises the question of whether Ramadan fasting affects sleep. In this review, we discuss the findings of research conducted to assess changes in sleep pattern, chronobiology, circadian rhythms, daytime sleepiness and function and sleep architecture during the month of Ramadan. Where applicable, these findings are compared with those obtained during experimental fasting.

Keywords: Ramadan; sleep; fasting; sleepiness; chronotype

(c) 2006 Blackwell Publishing Ltd

\section{INTRODUCTION}

Voluntary fasting, or the abstinence from food or drink or both, is practiced in many of the world's religions and cultures. Fasting during the holy month of Ramadan is the fourth pillar of Islam, and about 1.5 billion Muslims worldwide $(1,2)$ fast during Ramadan every year.

Researchers have long recognised that experimental fasting alters the sleep-wakefulness pattern in various species. For example, food deprivation has been shown to increase wakefulness and to markedly reduce rapid eye movement (REM) sleep (3-5). The results of experimental fasting, however, cannot be extrapolated to Ramadan fasting. In experimental fasting, the duration of each fasting episode is usually more prolonged than the intermittent fasting during Ramadan. Moreover, Ramadan fasting has unique characteristics. During every day of the month of Ramadan, Muslims abstain from food, drink and smoking between dawn and sunset. Sleep and wake patterns during Ramadan could be affected by the behavioural restrictions and regime imposed by the discipline of holy month.

The months in the Islamic (Hijra) year follow the lunar system. Each Hijra year contains 354 days, or 11 days fewer than the Gregorian year. As a result, the month of Ramadan

Correspondence to:

Dr Ahmed BaHammam, Associate Professor, Director Sleep Disorders Center, College of Medicine, Department of Medicine 38, King Saud University, PO Box 2925, Riyadh 11461, Saudi Arabia

Tel.: + 96614671521

Fax: + 96614672558

Email: ashammam@awalnet.net.sa occurs 11 days earlier every year, which means that Ramadan occurs during a different season every 9 years. The season during which Ramadan occurs affects the duration of each fast, because fasting hours are longer in summer than in winter. This, in turn, may affect sleep patterns, due to factors such as shorter nights and earlier dawns. In addition, climate may affect sleep. During the hot summer, many people resort to napping in the middle of the day, which may influence night sleep. Moreover, the geographical location may affect the duration of fasting. As one moves further from the equator, daytime becomes longer in summer and shorter in winter. Therefore, when studying sleep patterns during Ramadan, it is important to document the time of year, the location of the study and the times of dawn and sunset.

Several other features distinguish Ramadan fasting, including the sudden change in the circadian pattern of eating, whereby caloric intake increases at night, and the long duration of this practice ( 1 month), which may allow adaptation to the new regimen. In addition, there are changes in daynight activity patterns, such as rising for the predawn meal (suhur) and prayer, as well as attendant changes in lifestyle and habits that occur during Ramadan in some Islamic countries, such as opening of stores and shopping malls until late at night. All these factors indicate that the physiological and behavioural changes occurring during the month of Ramadan may be different from those in experimental fasting (6).

In Islamic countries, many individuals who fast associate Ramadan fasting with increased daytime sleepiness and decreased performance. This raises the question whether Ramadan fasting affects sleep. Although this question has attracted the attention of researchers in the past few years, to our knowledge, there has been no review of medical data addressing the question of sleep during Ramadan. We have 
therefore reviewed the findings of research conducted to assess changes in sleep pattern, chronobiology, circadian rhythms, daytime sleepiness and function and sleep architecture during the month of Ramadan. Where applicable, these results have been compared with those obtained during experimental fasting. References included in this article were obtained from journals on Medline as well as other peer review journals posted on the Internet.

\section{SLEEP PATTERN}

In some Islamic countries, working hours during Ramadan are shorter for those who fast. In such countries, fasting individuals are allowed to start work at 09:00-10:00 instead of the usual 07:00-08:00, a change which may affect sleep patterns. Therefore, it is important to report the working hours of the studied group, as this may affect the times they go to bed and wake up.

Surveys of sleep patterns during Ramadan have used different methods to assess bedtime, wake-up time, nighttime sleep duration and nap time and duration (7-12). Some of these surveys used a sleep diary, in which each participant recorded his or her bedtime, wake-up time, nighttime sleep duration and nap duration every day $(7,10,12)$. Mean values of these variables were then calculated for each participant. Other surveys required each participant to state the above variables the night before and during the preceding day $(8,9)$. All of these studies, conducted in three Islamic countries, consistently reported sudden significant delays in bedtime and wake-up time (Table 1). Interestingly, the delay in bedtime during Ramadan was reported even by non-Muslim residents in Saudi Arabia (KSA) (10). The delays in bedtime and wake-up time during Ramadan reported by fasting individuals in Islamic countries may be partially due to the delay in starting work during the month of Ramadan.

For nonfasting individuals, the time of starting work does not change; however, even for these individuals, bedtime is significantly delayed during Ramadan (10). These findings indicate that other factors may affect bedtime during Ramadan, including the delayed working hours for stores, shopping malls, and restaurants and the broadcasting of interesting TV programs until late at night. More than $60 \%$ of fasting individuals who stayed awake after 23:00 attributed their wakefulness to socialising with families and friends and watching TV $(7,10)$.

Conflicting results have been reported regarding sleep duration during Ramadan. When assessing sleep duration, however, it is important to include both nighttime sleep duration and daytime naps. In a sample of university students in Morocco, nighttime sleep duration was reported to drop significantly during Ramadan (8). A significant reduction in nighttime sleep duration during Ramadan was also observed in a sample of medical students in the United Arab Emirates, but this reduction was ameliorated by increasing the duration of daytime naps (9). In KSA, however, surveys of medical students (7) and employed healthy nonsmokers (10) between the ages of 25 and 55 years found no significant changes in nighttime sleep duration and total sleep time during the first and third weeks of Ramadan when compared with baseline (BL) values. While these studies of medical students and healthy workers $(7,10)$ found no significant change in the duration of naps or the percentage of subjects who napped before and during Ramadan, another study in university students in Morocco found a decrease in nap duration during Ramadan (8). Differences among the findings of these studies may be due to cultural and lifestyle differences in the study countries, for example, the start times of schools and work are delayed during Ramadan in some Islamic countries but not in others. Moreover, many of these studies (7-9) examined university students, a group of subjects prone to psychological and physical stresses due to the transitional nature of college life (13).

In summary, studies have demonstrated several modifications in sleep patterns during Ramadan, including sudden and significant delays in bedtime and wake-up time. However, conflicting results have been reported regarding total sleep time. Thus, larger scale studies, with reliable assessment of total sleep time among fasting individuals in Islamic and non-Islamic countries during Ramadan, are needed.

\section{CIRCADIAN RHYTHM}

Some of the changes associated with Ramadan may have some impact on the circadian rhythms and biological clock of

Table 1 Bedtime and wake-up time before and during Ramadan

\begin{tabular}{lccccc}
\hline & Baseline & Beginning of Ramadan & End of Ramadan & p-Value & Reference \\
\hline Bedtime, $\mathrm{h} \pm \mathrm{h}$ & $23.4 \pm 3.5$ & $2.8 \pm 2.9^{*}$ & $3.2 \pm 2.1^{*}$ & $<0.05$ & $7(\mathrm{mean} \pm \mathrm{SD})(n=56)$ \\
Wake-up time, $\mathrm{h} \pm \mathrm{h}$ & $6.6 \pm 0.9$ & $8.3 \pm 0.9^{*}$ & $8.95 \pm 0.4^{*}$ & $<0.001$ & \\
Bedtime, $\mathrm{h} \pm \mathrm{min}$ & $00: 36 \pm 14$ & $02: 06 \pm 16^{*}$ & $02: 36 \pm 19^{*}$ & $=0.001$ & $10(\mathrm{mean} \pm \mathrm{SE})(n=41)$ \\
Wake-up time, $\mathrm{h} \pm$ min & $06: 36 \pm 13$ & $08: 36 \pm 8^{*}$ & $08: 48 \pm 9^{*}$ & $=0.001$ & \\
Bedtime, $\mathrm{h} \pm \mathrm{min}$ & $23: 48 \pm 7$ & $00: 36 \pm 11$ & $00: 41 \pm 13$ & 0.0024 & $11(\mathrm{mean} \pm \mathrm{SE})(n=8)$ \\
Wake-up time, $\mathrm{h} \pm$ min & $08: 03 \pm 6$ & $08: 52 \pm 17$ & $09: 08 \pm 17$ & 0.016 & \\
\hline
\end{tabular}

*Significant compared with baseline. 
individuals during that month. Those who fast during Ramadan usually eat 2-3 meals per day: breakfast at sunset, dinner after night prayer (17:00-20:00) and a predawn meal. The eating of meals exclusively at night may therefore affect circadian rhythms during the fast. Body temperature normally follows a circadian rhythm, rising during the day and falling at night. In general, a falling body temperature induces sleep, whereas a rising temperature provokes wakefulness (7). When a rectal thermistor probe was used to record body temperature continuously for $24 \mathrm{~h}$ in six subjects during Ramadan, delays were observed in the occurrence of acrophase and bathyphase (i.e. the times at which the maximum- and minimum-calculated values, respectively, occur in the cycle), as well as a reduction in the amplitude (11). Another study of fasting during Ramadan found that oral temperature significantly decreased at 09:00, 11:00, 13:00 and 16:00 and significantly increased at 23:00 and 00:00 h (14). Similar changes in body temperature were not observed in another study that measured oral temperature using a high-precision medical thermometer at 08:00, 16:00 and 00:00 during the first and third weeks of Ramadan (12).

Melatonin is considered to be one of the best markers for circadian rhythm disruption $(14,15)$, as individual melatonin profiles are highly reproducible and are less subject to masking factors than are other rhythm markers like core temperature and cortisol (16). Thus, changes in the circadian pattern of body temperature during Ramadan may be accompanied by alterations in the circadian pattern of secretion of this hormone. In an assessment of melatonin levels from blood samples obtained every $4 \mathrm{~h}$, omitting the 02:00 point to avoid any sleep disturbances, before and on the 23rd day of Ramadan, a smaller delayed night peak and a flatter slope of serum concentrations of melatonin were observed during Ramadan (17). Using saliva samples obtained at three time points over a 24-h period (08:00,16:00 and 00:00), one week before Ramadan and on the 7th and 21st days of Ramadan, there were significant decreases in melatonin concentrations at 00:00 and 16:00 during Ramadan relative to BL (12). Nevertheless, melatonin profiles showed the same trend during Ramadan, but with a flatter slope (Fig. 1). In both studies, however, melatonin concentrations were not measured late at night, thus omitting any late peaks in melatonin concentration. Therefore, future studies should measure melatonin concentrations more frequently after midnight.

Melatonin concentration has been reported to decrease during fasting, even during short-term experimental fasting $(18,19)$. Although the exact mechanisms underlying the reduced levels of melatonin during fasting are not known, proposed mechanisms include increases in nocturnal cortisol levels during Ramadan $(20,21)$ and decreased melatonin synthesis secondary to decreased glucose provision (22). In support of the latter, malnourished rats have been found to have less melatonin in the pineal gland than normally fed

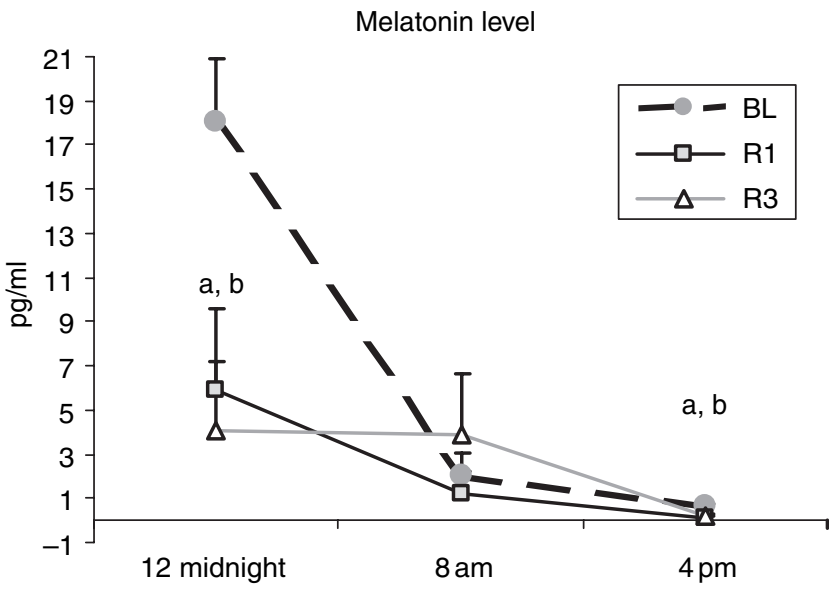

Figure 1 Circadian pattern of saliva concentrations of melatonin during baseline (BL), 1st week of Ramadan (R1) and 3rd week of Ramadan (R3). a and b indicate significant difference $(p<0.05)$ (a, BL vs. R1 and b, BL vs. R3) (12). Reproduced with permission

animals, an effect attributed to mild hypoglycaemia, which reduces the level of $\mathrm{N}$-acetyltransferase, an enzyme involved in the synthesis of melatonin, in starving rats (23). In fasting individuals, reduced melatonin level was found to be associated with mild hypoglycaemia, whereas glucose supplementation during fasting returned the decreased melatonin level to normal (19). This, however, may not explain the decreased melatonin level observed during Ramadan fasting, as hypoglycaemia is thought not to occur in fasting healthy individuals during the holy month (6). Another hypothesis proposed to explain reduced melatonin levels during fasting is the reduction in tryptophan, which is both an essential amino acid and a precursor of melatonin. This is unlikely, however, because glucose supplementation restored normal melatonin secretion (19).

Another way to assess circadian rhythms is to assess the chronobiology of fasting individuals. When we measured chronotype in fasting and nonfasting individuals using an abridged version of the Horne and Östberg questionnaire (morningness/eveningness test) (a questionnaire that establishes three behavioural categories: morning type, neither type and evening type) $(10,24)$, we observed a trend towards an increase in evening types among fasting individuals, both at the beginning and the end of Ramadan compared with BL. Similar findings were observed in fasting Muslims in Morocco (8). Interestingly, a change in chronotype was demonstrated in nonfasting non-Muslim residents in KSA during Ramadan (10). Among these individuals, there was a trend towards an increase in noon types and a decrease in morning types at both the beginning and end of Ramadan when compared with BL.

In summary, changes in the circadian rhythm and chronobiology have been demonstrated in fasting individuals during Ramadan. This is manifested as a change in the circadian 
pattern of body temperature, a decrease in the amplitude of the melatonin rhythm and a nocturnal increase in cortisol level.

\section{DAYTIME FUNCTION AND SLEEPINESS}

\section{Daytime Sleepiness and Alertness}

Studies have assessed daytime sleepiness subjectively (7-10, 12,25) using the Epworth sleepiness scale (ESS), which is a validated reliable sleep questionnaire that measures the general level of daytime sleepiness (26), and objectively using the multiple sleep latency test (MSLT) (12,25), with the latter test used to determine mean sleep latency during standardised daytime naps as an objective measure of daytime sleepiness; the presence/absence of REM sleep during the naps is also determined. Although some studies using the ESS have reported a significant increase in daytime sleepiness during the entire month of Ramadan $(7,8)$, others found no significant change $(9,10,12)$. When subjective alertness was assessed during Ramadan using a visual analogue scale, decreases in alertness were observed at 09:00 and 16:00, whereas an increase was observed at 23:00 (14). Other studies that used a 24-h time scale to determine the time of the day at which each participant felt most alert and most sleepy showed no differences between BL and Ramadan (Fig. 2; $7,10)$. These studies, however, recruited different groups of volunteers, with some including medical and university students, who may have had irregular sleep habits or a shortening of mean sleep length due to life constraints.

Two objective studies used the MSLT to evaluate sleepiness under controlled conditions (12,25). Because all participants had to be studied within a limited time (the month of Ramadan), there were constraints on the number of recruited volunteers, with each study including only eight subjects. In one study, increased daytime sleepiness at 10:00 and 12:00 was reported towards the end of Ramadan (25). However, this study used a portable, at home polysomnographyrecording device, which forced the operator to program the computer to end the test $20 \mathrm{~min}$ after the beginning of recording, regardless of sleep onset. Therefore, none of the participants slept long enough to progress to REM sleep. In the other study (12), a standard MSLT (27) was performed in the sleep laboratory, avoiding the limitation of the previous study. No differences in sleep latency, sleep onset

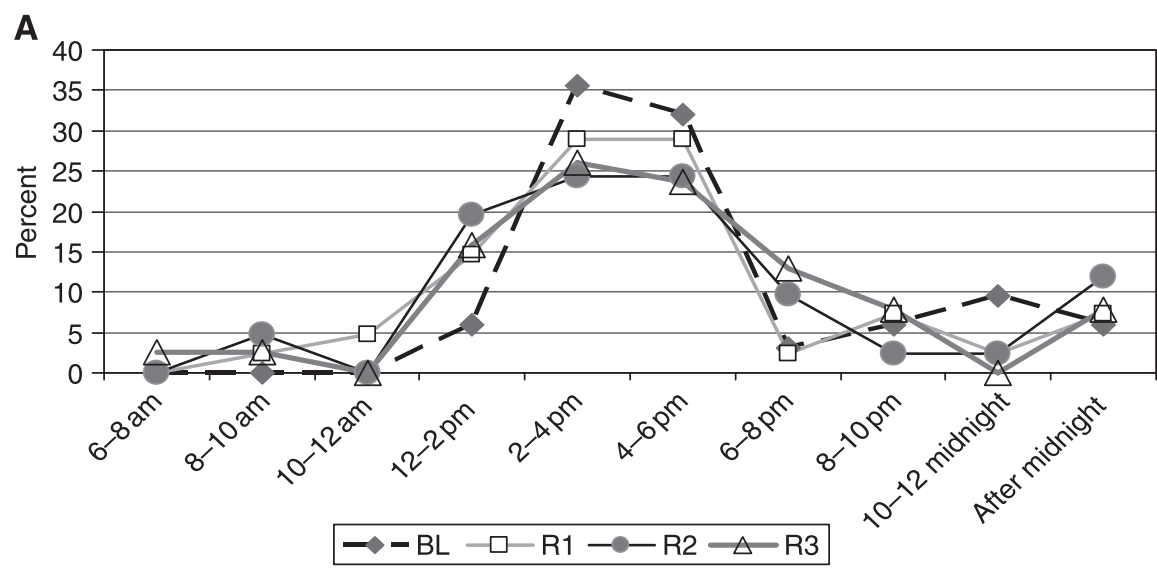

B

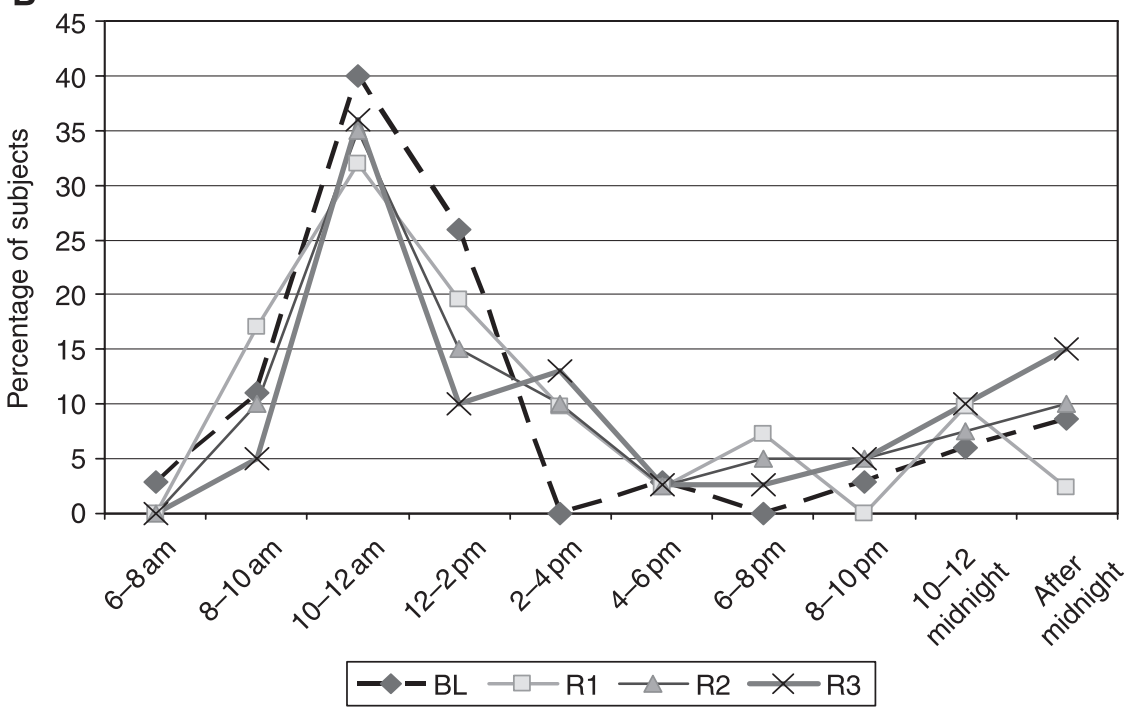

Figure 2 A) Time of the day when subjects felt most sleepy before and during the first 3 weeks of Ramadan. B) Time of the day when subjects felt most alert before and during the first 3 weeks of Ramadan. (BL, baseline data; R1, first week of Ramadan; R2, second week of Ramadan; R3, third week of Ramadan) (7). Reproduced with permission 
frequency and wake efficiency were observed between the first and third weeks of Ramadan and BL (12). Delta or slow wave activity during non-REM (NREM) sleep is considered a classic marker of the homeostatic process. In contrast, high levels of electro-encephalographic (EEG) alpha activity during wakefulness and NREM sleep may indicate a high level of alertness (28). Spectral analysis of the EEG activity during each nap showed no differences between Ramadan and BL (12).

\section{Daytime Function}

Daytime function during Ramadan has yet to be assessed properly under controlled conditions. During the first week of Ramadan, psychomotor performance indicators such as critical flicker fusion (29) and choice reaction time (14) have been reported to be impaired. The latter of these studies also found that sleep duration during Ramadan was $1 \mathrm{~h}$ less than BL (14). Even this modest sleep restriction may impact on daytime sleepiness and functioning (30). The percentage of medical students who reported falling asleep in class increased from $15 \%$ at $\mathrm{BL}$ to $36 \%$ during the first week of Ramadan (7). However, sleep deprivation or disruption cannot be ruled out as possible confounders in previous studies, as the participants' sleep duration and quality were not assessed objectively before and during Ramadan. An experimental study of individuals subjected to 1 week of controlled underfeeding and whose sleep was monitored by polysomnography found that during fasting, the study subjects showed increased daytime energy, concentration and emotional balance (18). Another study found a decrease in mood rating during Ramadan (14), but a second study did not observe this effect (31). Given that sudden delays in bedtime have been associated with adverse mood changes resembling those of depression $(32,33)$, the effect on mood of the sudden delay in bedtime during Ramadan requires further assessment. Although the percentage of subjects who exercise regularly dropped from $24 \%$ before Ramadan to $5 \%$ at the end of Ramadan (7), the reason for this decline is not clear.

In summary, the notion that Ramadan fasting affects daytime sleepiness and functioning is not supported by the available data. More research is needed to elucidate this issue, while controlling for potential confounders like sleep deprivation. Researchers should also remember that fatigue and alertness do not always parallel sleepiness (34). Sleepiness, alertness and fatigue may be symptoms of poor sleep, but testing by independent evaluations is required.

\section{POLYSOMNOGRAPHY AND SLEEP ARCHITECTURE}

Two studies have assessed sleep architecture during Ramadan using polysomnography: one used ambulatory 8-channel unattended recording at home (11), and the second used full attended in-laboratory polysomnographic recording (12) (Table 2). Both studies demonstrated a significant reduction in REM sleep towards the end of Ramadan, which contrasts with findings of increased REM sleep during experimental fasting in humans (18). However, the fasting pattern in the experimental study was controlled underfeeding rather than intermittent fasting. An experimental study conducted on piglets demonstrated that REM sleep did not occur after $18 \mathrm{~h}$ of fasting but recurred after feeding (35). The reduction in REM sleep during fasting may be due to a nocturnal rise in cortisol and insulin (36-38). Nocturnal body temperature has been reported to increase during sleep $(11,14)$. Because REM sleep is inversely proportional to core temperature $(39,40)$, a nocturnal increase in temperature would be expected to decrease REM sleep $(41,42)$. Another possible explanation for the reduction in REM sleep during Ramadan is the interruption of sleep for the predawn meal during the early morning hours, the time period in which a larger amount of REM sleep usually occurs.

Conflicting results have been reported with regard to sleep latency and total sleep time. While one study reported a

Table 2 Polysomnographic characteristics before and during Ramadan

\begin{tabular}{|c|c|c|c|c|c|}
\hline & Baseline & Beginning of Ramadan & End of Ramadan & p-Value & Reference \\
\hline TIB (min) & $440.2 \pm 9.3$ & $472.6 \pm 10.9$ & $476.3 \pm 4.8$ & NS & $12($ mean $\pm S E)(n=8)$ \\
\hline TST (min) & $361.7 \pm 39$ & $379.0 \pm 36.0$ & $378.0 \pm 35.0$ & NS & \\
\hline Sleep latency (min) & $35.0 \pm 12.0$ & $30.4 \pm 17$ & $18.5 \pm 10^{*}$ & 0.05 & \\
\hline REM percentage (TST) & $24.1 \pm 3.9$ & $21.5 \pm 4.2$ & $10.2 \pm 5.0^{*}$ & 0.003 & \\
\hline Arousal index & $19.9 \pm 6.8$ & $18.1 \pm 10.9$ & $19.0 \pm 11.0$ & NS & \\
\hline Stage shifts & $94.0 \pm 23.5$ & $95.0 \pm 23.8$ & $101.8 \pm 22.0$ & NS & \\
\hline TIB (min) & $454 \pm 4.7$ & $406 \pm 11.9^{*}$ & $422 \pm 14.9$ & $<0.01$ & $11($ mean $\pm S E)(n=8)$ \\
\hline TST (min) & $422 \pm 9.0$ & $381 \pm 11.6^{*}$ & $383 \pm 16.7^{*}$ & $<0.05$ & \\
\hline Sleep latency (min) & $19.2 \pm 2.6$ & $60.5 \pm 10.6^{*}$ & $58.1 \pm 14.8^{*}$ & $<0.01$ & \\
\hline REM sleep (min) & $94.3 \pm 5.2$ & $88.5 \pm 6.6$ & $74.7 \pm 5.3^{*}$ & $<0.01$ & \\
\hline
\end{tabular}

NS, nonsignificant; TIB, time in bed; TST, total sleep time. *Significant compared with baseline. 
significant drop in sleep latency at the end of Ramadan and no change in total sleep time (12), a second study (11) reported a significant increase in sleep latency and a significant reduction in total sleep time. The discrepancy between these results may be due to the amounts of time between dinner and bedtime. In one study (11), the difference between dinnertime and bedtime was $1 \mathrm{~h}$ (dinner was served at 22:30 and polysomnographic recording started at 23:30), whereas, in the second study (12), the difference was $3 \mathrm{~h}$ (dinner was served at 21:00 and recording started at 00:00). Because late dinner may affect nocturnal sleep, future research should consider the relation between dinnertime during Ramadan and nocturnal sleep.

No significant differences were demonstrated between Ramadan and BL with regard to NREM sleep stages, arousal index, stage shifts and cardio-respiratory parameters (12). Using an experimental model of fasting, significant reductions were observed in the number of arousals and arousal index, as well as a significant reduction in periodic leg movement (PLM) during fasting (18). Although no differences in PLM before and during Ramadan have been reported (12), the mean PLM in the latter (12) was not high compared with that in the former (18).

In summary, a reduction in the proportion of REM sleep has been reported during Ramadan, although these studies have shown conflicting results with regard to sleep latency and total sleep time. Larger studies that control for different confounders, such as the time of the dinner meal, are needed.

\section{REFERENCES}

1 The World Factbook 2005. ISSN 1553-8133 (updated periodically throughout the year; cited 5 September 2005. Available from http://www.odci.gov/cia/publications/factbook

2 Muslim population worldwide. (updated 21 December 2004; cited 5 September 2005). Available from http://www. islamicpopulation.com

3 Borbely AA. Sleep in the rat during food deprivation and subsequent restitution of food. Brain Res 1977; 124: 457-71.

4 Danguir J, Nicolaidis S. Dependence of sleep on nutrients' availability. Physiol Behav 1979; 22: 735-40.

5 Rashotte ME, Pastukhov IF, Poliakov EL, Henderson RP. Vigilance states and body temperature during the circadian cycle in fed and fasted pigeons (Columba livia). Am J Physiol 1998; 275 (5 Part 2): R1690-1702.

6 Azizi F. Research in Islamic fasting and health. Ann Saudi Med 2002; 22: 186-91.

7 Bahammam A. Sleep pattern, daytime sleepiness, and eating habits during the month of Ramadan. Sleep Hypnosis 2003; 5: 165-74.

8 Taoudi Benchekroun M, Roky R, Toufiq J, Benaji B, Hakkou F. Epidemiological study: chronotype and daytime sleepiness before and during Ramadan. Therapie 1999; 54: 567-72.
9 Margolis SA, Reed RL. Effect of religious practices of Ramadan on sleep and perceived sleepiness of medical students. Teach Learn Med 2004; 16: 145-9.

10 Bahammam A. Assessment of sleep patterns, daytime sleepiness, and chronotype during Ramadan in fasting and nonfasting individuals. Saudi Med J 2005; 26: 616-22.

11 Roky R, Chapotot F, Hakkou F, Benchekroun MT, Buguet A. Sleep during Ramadan intermittent fasting. I Sleep Res 2001; 10: 319-27.

12 Bahammam A. Effect of fasting during Ramadan on sleep architecture, daytime sleepiness and sleep pattern. Sleep Biol Rhythm 2004; 2: 135-43.

13 Ross SE, Niebling BC, Heckert TM. Sources of stress among college students. College Student J 1999; 33: 312-8.

14 Roky R, Iraki L, HajKhlifa R, Lakhdar Ghazal N, Hakkou F. Daytime alertness, mood, psychomotor performances, and oral temperature during Ramadan intermittent fasting. Ann Nutr Metab 2000; 44: 101-7.

15 Miles A, Thomas R. Melatonin - a diagnostic marker in laboratory medicine? In: Miles A, Philbrick DRS, Thompson C, eds. Melatonin: Clinical Perspectives. Oxford, UK: Oxford University Press, 1988: pp. 253-79.

16 Arendt J. Melatonin and the Mammalian Pineal Gland. London, UK: Chapman \& Hall, 1995.

17 Bogdan A, Bouchareb B, Touitou Y. Ramadan fasting alters endocrine and neuroendocrine circadian patterns. Meal-time as a synchronizer in humans? Life Sci 2001; 68: 1607-15.

18 Michalsen A, Schlegel F, Rodenbeck A et al. Effects of shortterm modified fasting on sleep patterns and daytime vigilance in non-obese subjects: results of a pilot study. Ann Nutr Metab 2003; 47: 194-200.

19 Röjdmark S, Wetterberg L. Short-term fasting inhibits the nocturnal melatonin secretion in healthy man. Clin Endocrinol 1989; 30: 451-7.

20 Al-Hadramy MS, Zawawi TH, Abdelwahab SM. Altered cortisol levels in relation to Ramadan. Eur J Clin Nutr 1988; 42: 359-62.

21 Sliman NA, Ajlouni KS, Faisal K. Effect of fasting on some blood hormones in healthy Muslim males. Mu'tah J Res Stud 1993; 8: 91-109.

22 Chik CL, Ho AK, Brown GM. Effects of food restriction on 24-h serum and pineal melatonin content in male rats. Acta Endocrinol 1987; 115: 507-13.

23 Welker HA, Vollrath L. The effects of a number of short-term exogenous stimuli on pineal serotonin- $N$-acetyltransferase activity in rats. J Neural Transm 1984; 59: 69-80.

24 Horne JA, Östberg O. A self-assessment questionnaire to determine morningness-eveningness in human circadian rhythms. Int J Chronobiol 1976; 4: 97-110.

25 Roky R, Chapotot F, Benchekroun MT et al. Daytime sleepiness during Ramadan intermittent fasting: polysomnographic and quantitative waking EEG study. J Sleep Res 2003; 12: 95-101.

26 Johns MW. A new method for measuring daytime sleepiness: the Epworth sleepiness Scale. Sleep 1991; 14: 540-5.

27 Thorpy MJ. The clinical use of the Multiple Sleep Latency Test. The Standards of Practice Committee of the American Sleep Disorders Association. Sleep 1992; 15: 268-76. 
28 Cajochen C, Dijk DJ. Electroencephalographic activity during wakefulness, rapid eye movement and non-rapid eye movement sleep in humans: comparison of their circadian and homeostatic modulation. Sleep Biol Rhythms 2003; 2: 85-95.

29 Ali MR, Amir T. Effects of fasting on visual flicker fusion. Percept Mot Skills 1989; 69: 627-31.

30 Devoto A, Lucidi F, Violani C, Bertini M. Effects of different sleep reductions on daytime sleepiness. Sleep 1999; 22: 336-43.

31 Daradkeh TK. Parasuicide during Ramadan in Jordan. Acta Psychiatr Scand 1992; 86: 253-4.

32 Totterdell P, Reynolds S, Parkinson B, Briner RB. Associations of sleep with everyday mood, minor symptoms and social interaction experience. Sleep 1994; 17: 466-75.

33 David MM, MacLean AW, Knowles JB, Coulter ME. Rapid eye movement latency and mood following a delay of bedtime in healthy subjects: Do the effects mimic changes in depressive illness? Acta Psychiatr Scand 1991; 84: 33-9.

34 Thorpy MJ. Which clinical conditions are responsible for impaired alertness? Sleep Med 2005; 6: S13-S20.

35 Kotrbacek V, Schweigel M, Honig Z. The effect of short-term fasting on sleep in pigs. Vet Med (Praha) 1990; 35: 547-52.

36 Sangiah S, Caldwell DF. Reduction of rapid eye movement (REM) sleep by glucose alone or glucose and insulin in rats. Life Sci 1988; 42: 1425-9.
37 Born J, Spath-Schwalbe E, Schwakenhofer H, Kern W, Fehm HL. Influences of corticotropin-releasing hormone, adrenocorticotropin, and cortisol on sleep in normal man. J Clin Endocrinol Metab 1989; 68: 904-11.

38 Krieger DT, Glick SM. Sleep EEG. stages and plasma growth hormone concentration in states of endogenous and exogenous hypercortisolemia or ACTH elevation. J Clin Endocrinol Metab 1974; 39: 986-1000.

39 Jouvet M, Buda C, Debilly G, Dittmar A, Sastre JP. Central temperature is the principal factor of regulation of paradoxical sleep in pontile cats. C R Acad Sci III 1988; 306: 69-73.

40 Krueger JM, Takahashi $S$. Thermoregulation and sleep. Closely linked but separable. Ann NY Acad Sci 1997; 813: 281-6.

41 Libert JP, Di Nisi J, Fukuda H, Muzet A, Ehrhart J, Amoros C. Effect of continuous heat exposure on sleep stages in humans. Sleep 1988; 11: 195-209.

42 Haskell EH, Palca JW, Walker JM, Berger RJ, Heller HC. Metabolism and thermoregulation during stages of sleep in humans exposed to heat and cold. J Appl Physiol 1981; 51: 948-54.

Paper received August 2005, accepted December 2005 